Revue d'histoire de l'Amérique française

BEVUE D.HISTOIRE DE L'AMÉRIQUE FRANÇAISE

\title{
BROOKS, Stephen et Alain G. GAGNON, Les spécialistes des sciences sociales et la politique au Canada. Entre l'ordre des clercs et l'avant-garde (Montréal, Boréal, 1994), 226 p.
}

\section{Marcel Fournier}

Volume 48, numéro 4, printemps 1995

URI : https://id.erudit.org/iderudit/305366ar

DOI : https://doi.org/10.7202/305366ar

Aller au sommaire du numéro

Éditeur(s)

Institut d'histoire de l'Amérique française

ISSN

0035-2357 (imprimé)

1492-1383 (numérique)

Découvrir la revue

Citer ce compte rendu

Fournier, M. (1995). Compte rendu de [BROOKS, Stephen et Alain G. GAGNON, Les spécialistes des sciences sociales et la politique au Canada. Entre l'ordre des clercs et l'avant-garde (Montréal, Boréal, 1994), 226 p.] Revue d'histoire de l'Amérique française, 48(4), 544-548. https://doi.org/10.7202/305366ar d'utilisation que vous pouvez consulter en ligne. 
BROOKS, Stephen et Alain G. GAGNON, Les spécialistes des sciences sociales et la politique au Canada. Entre l'ordre des clercs et l'avantgarde (Montréal, Boréal, 1994), 226 p.

D'abord publié en anglais chez McGill-Queen's University Press en 1988, l'ouvrage de Stephen Brooks et Alain G. Gagnon, tous deux professeurs de science politique, l'un à l'Université Windsor et l'autre à l'Université McGill, s'inscrit dans une réflexion sur le rôle politique des intellectuels. Dans l'introduction, les auteurs font le tour de la question, présentent 
quatre interprétations de la position de classe et du rôle politique des intellec tuels (légitimation du pouvoir, potentiel révolutionnaire, membres de la classe dirigeante et leaders, position critique) et, faisant appel aux concepts de légitimation et de changement social, tentent de concilier ces diverses interprétations. Comme l'indique le sous-titre de leur ouvrage, tout se ramène à l'opposition entre, d'un côté, les tenants de l'ordre ou les clercs - des chiens de garde, aurait dit Paul Nizan - et de l'autre, les tenants du changement social ou les avant-gardistes.

Fondée sur des sources primaires et surtout secondaires, l'étude se limite à un sous-ensemble des intellectuels, les spécialistes en sciences sociales, et a, comme point de départ, la Seconde Guerre mondiale. S'agissant du Canada, la grande question est évidemment celle des différences entre les intellectuels canadiens-français et les intellectuels canadiens-anglais. D'ailleurs, l'ouvrage est composé, comme on pouvait s'y attendre, de deux grandes parties, la première consacrée au Québec et la seconde, au Canada anglais. Et dans un cas comme dans l'autre, on a établi, pour l'ensemble des disciplines en sciences sociales, la même périodisation: 1) des années 1940 aux années 1960, 2) la décennie des années 1960, et enfin 3) la période de 1970 à 1986.

Pour le Québec, cette périodisation renvoie à trois étapes du développement des sciences sociales: 1) l'institutionnalisation et l'élaboration d'une contre-idéologie, 2) la maturation et l'engagement, et 3) la fragmentation idéologique et la fin du consensus nationaliste. On passe, si on utilise le concept de légitimité, de la lutte pour la légitimité à la légitimité en marche pour arriver à la légitimité en crise (p. 172-174). Qu'est-ce à dire? Pour la première période, les auteurs présentent une bonne synthèse des travaux portant sur l'institutionnalisation des sciences sociales: le rôle du père Lévesque, l'opposition au régime duplessiste et la Révolution tranquille. Rien de très nouveau. Si elle avait été plus approfondie, l'analyse aurait permis de mettre en évidence quelques principes d'opposition, par exemple entre les universités Laval et de Montréal: face au père Lévesque qui était fédéraliste (et qui fut membre de la commission Massey), il y avait les économistes, Esdras Minville et François-Albert Angers qui étaient nationalistes (et qui furent, à un moment donné, «experts» pour le gouvernement de Duplessis). Sans parler des historiens, membres de l' «École de Montréal». Tous les spécialistes en sciences sociales n'exercèrent pas le rôle d' «avant-garde». Il est donc inexact de tous les identifier à la Révolution tranquille et abusif de laisser entendre qu'«ils ont été au nombre des principaux bénéficiaires de cette mutation» (p. 175).

Faut-il parler de la deuxième période comme d'une période de «maturation»? L'expression est vague à souhait: les sciences sociales parviennentelles alors au terme de leur développement? Cette «maturation» correspond, selon les auteurs, à la phase de «nationalisation» des sciences sociales, de spécialisation croissante de chacune des disciplines et, enfin, de dissociation de la fonction universitaire de son pendant social. Mais paradoxalement, cette phase, qui voit des spécialistes en sciences sociales (Guy Coulombe, Maurice Lamontagne, etc.) accéder à des postes de pouvoir dans les appareils 
administratifs tant au provincial qu'au fédéral, est aussi celle de la radicalisation de l'engagement social des universitaires, dont les expressions privilégiées sont la participation à des expériences d'animation sociale (en région et en ville), au mouvement syndical et à des revues, tel Parti pris (qui est animée non seulement par des spécialistes en sciences sociales, mais aussi par des littéraires et des philosophes). On aurait pu aussi parler de la revue Socialisme, fondée en 1964 par Jacques Dofny et Marcel Rioux.

À cette période fort agitée succéda une période de «refroidissement» (qui conduisit, disent les auteurs, à une «hibernation temporaire»): vint ensuite la «fragmentation idéologique» et ce fut, dans les années 1970-1986, la «fin du consensus nationaliste». Comme s'il y avait eu auparavant un quelconque consensus parmi les spécialistes en sciences sociales! Qu'y avait-il de commun entre un Marcel Rioux et un abbé Norbert Lacoste? Comment peuton dire que «les sciences sociales évoluaient dans le sens d'une diversité idéologique croissante»? Afin d'illustrer cette diversité croissante, les auteurs donnent trois exemples: l'étude du Québec dans une perspective néomarxiste, la redécouverte des régions et, enfin, le constat de la diversité ethnoculturelle de la société québécoise. Le portrait est fort incomplet et néglige de prendre en considération les effets de deux «révolutions» - la révolution culturelle (contre-culture, etc.) et le féminisme - sur les sciences sociales.

Les auteurs constatent, par ailleurs, avec un certain regret semble-t-il, la métamorphose de l'UQAM, le développement d'un certain conservatisme et la baisse de popularité des sciences sociales, qui se voient, dans les universités, dépassées par les sciences administratives et commerciales. C'est la «révolution des affaires», et avec le retour de Robert Bourassa au pouvoir en 1985, la «relève de la garde». Les auteurs n'excluent cependant pas l'hypothèse d'une «politisation accrue» et d'une éventuelle mobilisation «sous la bannière d'un nationalisme renaissant».

$\mathrm{Si}$, pour plusieurs d'entre eux, les spécialistes québécois peuvent être identifiés d'avant-gardistes, c'est en quelque sorte le contraire pour leurs collègues canadiens-anglais, qui apparaissent le plus souvent comme des clercs: ces spécialistes, en particulier les économistes, ont réussi à se faire reconnaître rapidement comme experts et «à pénétrer profondément le système d'élaboration des politiques gouvernementales» (p. 174). L'étude se limite principalement aux relations entre les sciences sociales et le gouvernement fédéral. Toute périodisation du développement des sciences sociales au Canada anglais qui ne tienne compte que du rôle politique apparaît dès lors difficile. Les trois périodes que tentent d'identifier Brooks et Gagnon sont les suivantes: 1) Le nouvel ordre des clercs (1945-1963), 2) L'expansion des sciences sociales et la dissidence nationaliste (1963-1971) et 3) Une avant-garde dans l'impasse et la consolidation du rôle d'expert (1971-1986).

Du côté du Canada anglais, les dissidents intellectuels n'ont jamais eu une revue aussi influente que Cité libre; ils ont, par ailleurs, été «impuissants» à formuler une critique de classes de la société et ils n'ont réussi à avoir une réelle influence ni sur le mouvement ouvrier canadien ni sur le 
NPD. Leur seule véritable dissidence fut nationaliste, à un moment où les universités canadiennes connurent un essor spectaculaire dans les années 1960: cette dissidence mettait en question certes la dépendance (culturelle et économique) du Canada à l'égard des États-Unis, mais aussi la proportion élevée de non-Canadiens dans les universités canadiennes (et indirectement l'influence américaine sur les sciences sociales). De plus s'est développée ce que les auteurs appellent «l'aile gauche des spécialistes en sciences sociales» c'est-à-dire l'apparition d'une nouvelle économie politique d'inspiration marxiste et la création de la revue Studies of Political Economy. Mais contrairement à ce qui s'est passé au Québec, les intellectuels de gauche du Canada anglais se sont révélés «incapables d'élaborer une analyse de la société qui exprimât les intérêts d'une classe montante» (p. 158).

En conclusion, les auteurs résument leur étude et soulèvent la question incontournable des différences entre le Québec et le Canada anglais. La tentation est évidemment grande d'attribuer à des «différences culturelles les relations divergentes qu'entretiennent avec la politique les spécialistes des sciences sociales du Canada anglais et ceux du Québec francophone» (p. 181). Brooks et Gagnon rejettent cette interprétation: les «facteurs essentiels» sont d'ordre historique et politique. Tout s'explique par la Conquête et par «l'assujettissement social et économique du Canada français» qu'elle a entraîné. Puis il y a eu, au Québec, une «constellation de développements sociaux et économiques» (p. 184), phénomène inconnu au Canada anglais, qui a favorisé ce qu'on pourrait appeler une «collusion» entre les intérêts des spécialistes en sciences sociales et ceux d'une «nouvelle classe moyenne montante».

Les liens entre les intellectuels et les classes sociales ne sont ni aussi directs ni aussi simples. Il y aurait peut-être lieu d'emprunter la problématique de Pierre Bourdieu (que les auteurs ne citent pas) et d'introduire la notion de champ intellectuel. Y a-t-il, pour les sciences sociales, un champ intellectuel canadien? Comment se structure l'ensemble des positions occupées par des spécialistes en sciences sociales? Quels sont les intellectuels qui occupent des positions supérieures et dominantes? Et si la politique est si importante pour certains d'entre eux, c'est peut-être qu'elle représente une voie d'accès à de telles positions...

En divisant leur étude en deux grandes parties, les auteurs évitent de se poser de telles questions. Il est souvent difficile de faire autrement... mais l'analyse risque d'être incomplète, quelque peu tronquée. Que dire du rôle d'organismes tels le Conseil des Arts et le Conseil de recherches en sciences humaines du Canada? Et puisque pour le Canada anglais tout semble tourner autour d'Ottawa, on voit mal le rôle que jouent les spécialistes en sciences sociales auprès des divers gouvernements provinciaux. Enfin, que dire de l'action d'intellectuels franco-canadiens qui, tels les Albert et Raymond Breton, ont fait leur carrière à l'Université de Toronto? ou d'Hubert Guindon, franco-ontarien d'origine, qui, après ses études universitaires, est venu s'installer au Québec? Faut-il situer McGill et Concordia du côté du Québec ou du Canada anglais? Lui-même professeur à l'Université McGill, Alain G. 
Gagnon n'analyse que rapidement le rôle politique de ses collègues, par exemple Maurice Pinard.

Il est vrai que dans son ensemble, cette étude sur les sciences sociales au Canada est rapide (quelque deux cents pages) et qu'elle apparaît parfois superficielle. Le projet était, comme le soulignent les auteurs, ambitieux. Le mérite de leur démarche est évidemment d'être comparative et de permettre aux lecteurs de langue française, qui ont maintenant accès à la traduction française, de mieux connaître et comprendre le milieu intellectuel et universitaire canadien-anglais. 